\title{
Identification of regulatory elements in the human adipose most abundant gene transcript-1 (apM-1) promoter: role of SP1/SP3 and TNF- $\alpha$ as regulatory pathways
}

\author{
N. Barth ${ }^{2}$, T. Langmann 3 , J. Schölmerich ${ }^{2}$, G. Schmitz ${ }^{3}$, A. Schäffler ${ }^{1,2}$ \\ ${ }^{1}$ Department of Internal Medicine I, University of Regensburg, Regensburg, Germany \\ ${ }^{2}$ Department of Internal Medicine I, University of Regensburg, Germany \\ ${ }^{3}$ Institute of Clinical Chemistry and Laboratory Medicine, University of Regensburg, Germany
}

\section{Diabetologia (2002) 45:1425-1433}

Table 1 was missing in this article.

DOI $10.1007 / \mathrm{s} 00125-003-1278-2$

Published online: 20 November 2003

(C) Springer-Verlag 2003

The online version of the original article can be found at http://dx.doi.org/10.1007/s00125-002-0895-5

A. Schäffler (四), Department of Internal Medicine I, University of Regensburg, 93042 Regensburg, Germany

E-mail: andreas.schaeffler@ klinik.uni-regensburg.de

Table 1. Oligonucleotide sequences used for electrophoretic mobility shift assays

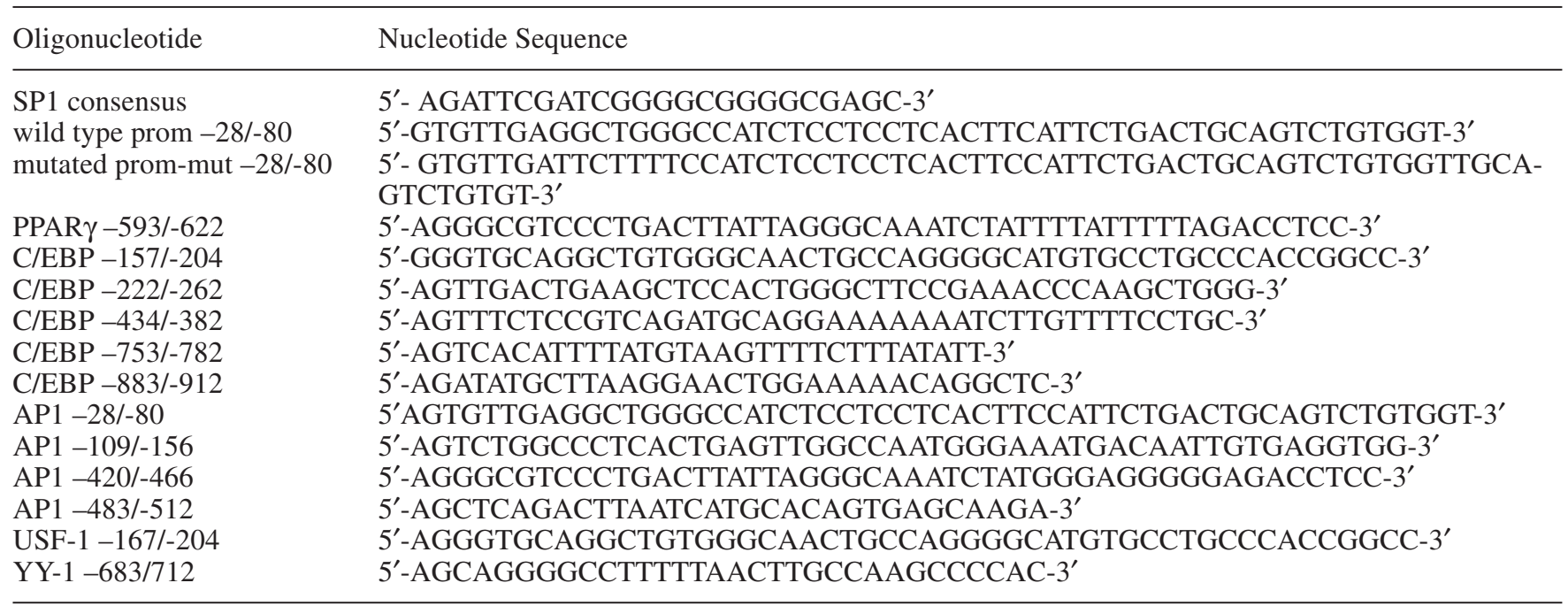

